\title{
Network programming technique for unit commitment
}

\author{
R Zhu and C Fu \\ Electric Power Research Institute, Qinghe, \\ Beijing 100085. People's Republic of China
}

\section{S Rahman}

Department of Electrical Engineering. Virginia Tech, Blacksburg, VA 24061, USA
In this paper, a new approach for short-term unit commitment is proposed. The unit commitment problem is formulated as a network problem and solved by a network programming technique. The proposed algorithm has been tested on a system of up to 61 units to be scheduled over 24 hours. Experimental results show that this algorithm can obtain a satisfactory solution in 46.51 seconds on a $V A X 11 / 750$ computer.

Keywords: unit commitment, network programming, economic dispatch

\section{Introduction}

Unit commitment is one of the most important activities in the operation of a power system. Optimal unit commitment determines when to start and when to shut down units so that the total cost is minimal and all constraints are satisfied. Many methods have been proposed to solve this problem. Baldwin ${ }^{1}$ proposed a priority order method based on a priority list of units. This is the earliest and simplest approach to determine unit schedules. Garver ${ }^{2}$ has used an integer programming technique to determine the unit commitment schedule. Muckstadt ${ }^{3}$ has formulated this problem as a mixedinteger programming problem. Other methods such as dynamic programming (DP) approach ${ }^{4,5}$, Lagrangian relaxation method ${ }^{6}$ and branch-and-bound method ${ }^{7}$ have been extensively surveyed. Recently, expert systems and neural networks have been used in solving the unit commitment problem ${ }^{8,9}$.

This paper describes an algorithm for short-term unit commitment and shows how to solve this problem via

Received 3 September 1993; revised 9 February 1994; accepted 19 April 1994 network programming. This approach models the unit commitment problem as a network shortest path problem and solves the problem using a network optimization algorithm. This method is simple, sufficient and fast because of exploiting network structures. In this network, nodes represent unit combinations and are connected by arcs. The arc cost includes start cost and unit production cost. Two nodes are designated as the source and the sink. When the shortest path connecting the source to the sink is found, the optimal unit commitment schedule observed with various operating constraints can be easily obtained.

To demonstrate the effectiveness of the proposed method, case studies are performed on a 61 unit system. For comparison, load profiles are also scheduled by the DP algorithm. The results indicate the validity of this algorithm.

\section{Network definitions}

The structure of a network model is defined by nodes and arcs. A node $i$ is an element of the list of nodes, $N=[1,2, \ldots, i, \ldots, n]$. An arc may be defined by an ordered pair of nodes $(i, j)$ or as an element, say element $k$, of the list of arcs. An arc $(i, j)$ is said to originate at node $i$ and terminate at node $j$. A node represents an event and an arc represents the specified relationships between two events. This representation is useful for modelling a wide range of physical and conceptual situations and provides a visual display of the network. Nodes are symbolized by circles containing the node index. An arc is shown by a directed line segment connecting its origin and terminal nodes. Figure 1 is a graphical model showing the structure characteristics of an example network.

The network models are described by parameters such as arc cost, arc capacity, a lower bound and arc gain. These parameters are used to solve the network programming problems. 


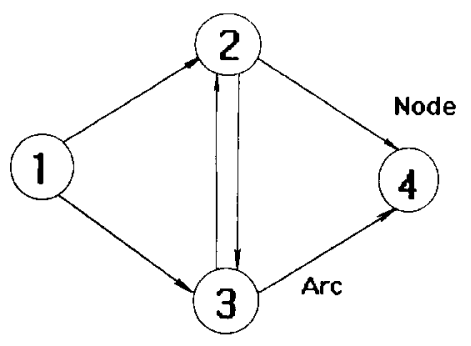

Figure 1. Example network

\section{Problem formulation}

In this study, a second-order polynomial is used to describe the fuel cost against the generation curve of each thermal unit.

$$
f(p)=a p^{2}+b p+c
$$

The start up cost of each thermal unit is an exponential function of the time that unit has been off:

$$
S=S_{0}\left(1-\mathrm{e}^{-t / \tau}\right)+\psi
$$

The following constraints are to be met for optimal solution.

System generation and demand must balance:

$$
\sum_{i=1}^{N} P_{i}(k)=D(k) \quad k=1, \ldots, T
$$

Generation limit of each unit:

$$
P_{i \min } \leqslant P_{i}(k) \leqslant P_{i \max } \quad i=1, \ldots, N ; k=1, \ldots, T
$$

Spinning reserve constraints:

$$
\sum_{i=1}^{N} P_{i \min } X_{i}(k) \leqslant D(k)+R(k) \leqslant \sum_{i=1}^{N} P_{i \max } X_{i}(k)
$$

Minimum up-time of each unit:

$$
\left(t_{i(k-1)}^{\mathrm{on}}-T_{i}^{\mathrm{n}}\right)\left(X_{i(k-1)}-X_{i(k)}\right) \geqslant 0
$$

Minimum down-time of each unit:

$$
\left(t_{i(k-1)}^{\mathrm{off}}-T_{i}^{\mathrm{fff}}\right)\left(X_{i(k)}-X_{i(k-1)}\right) \geqslant 0
$$

The objective function for the unit commitment problem can be formulated as:

$$
\begin{aligned}
& \min \sum_{k=1}^{T} \sum_{i=1}^{N}\left[f\left(P_{i(k)}\right) X_{i(k)}+S_{i}\left(t_{i(k-1)}^{\text {off }}\right)\right] \\
& S_{i}\left(t_{i(k-1)}^{\text {off }}\right) \\
& =\max \left[0,\left(X_{i(k)}-X_{i(k-1)}\right)\left(K+S_{0}\left(1-\exp \left[-t_{i(k-1)}^{\text {off }}\right) / T\right]\right)\right]
\end{aligned}
$$

Subject to constraints in equations (3)-(7). Where: $a, b, c$ cost coefficients of thermal unit

$\psi \quad$ fuel cost required to start the turbine of unit

$S_{0} \quad$ fuel cost required to start cold unit boiler

$\tau \quad$ cooling time constant

$T$ number of hours in the unit commitment horizon

$N$ number of available generation units

$k$ hour index in the commitment horizon

$i \quad$ unit index

$P_{i \min } \quad$ minimum generation limit of unit $i$

$P_{i \max } \quad$ maximum generation limit of unit $i$

$D(k) \quad$ system demand at hour $k$ in $\mathrm{MW}$
$X_{i}(k) \quad$ '0-1' decision variable of unit $i$ at hour $k$. ' 1 ' indicates on-line, ' 0 ' indicates off-line

$R(k) \quad$ system spinning reserve requirement at hour $k$ in MW

$P_{i}(k) \quad$ generation level of unit $i$ in period $k$

$T_{i}^{\text {on }} \quad$ minimum on-time of unit $i$

$T_{i}^{\text {off }} \quad$ minimum off-time of unit $i$

$t_{i(k)}^{i \text { n }} \quad$ continuous up-time of unit $i$ including period $k$

$t_{i(k)}^{\text {off }} \quad$ continuous down-time of unit $i$, including period $k$

The optimal unit commitment problem is a zero-one non-linear optimization problem.

\section{Description of the proposed approach}

The proposed technique is based on network programming and can be divided into two steps as follows:

(1) generating a network model for unit commitment; and

(2) choosing an algorithm to solve this problem and developing a computer program of the chosen algorithm that can solve the problem efficiently.

Each of these steps is discussed in detail below.

\section{IV.1 Defining the unit commitment network}

In the unit commitment network, a node represents a combination, an arc represents the transition from one combination at a given hour to a combination at the next hour. Each hour represents one stage. All the nodes that belong to the same stage are put on a perpendicular line and nodes belonging to different stages are set sequentially. The initial combination of units is designated as the source node, and finally, a dummy node is designated as the sink node. This network model is illustrated in Figure 2.

The arc cost $a(i, j)$ associated with a transition from combination $i$ at hour $k$ to combination $j$ at hour $k+1$ is calculated by the following formula:

$$
a(i, j)= \begin{cases}F(i)+S(i, j) & \alpha \\ \infty & \beta\end{cases}
$$

$\alpha$ for the case where all constraints are met, and $\beta$ for one or more constraints are violated, where

$F(i) \quad$ fuel cost of combination $i$ at hour $k$. In this paper, $F(i)$ is calculated by economic dispatch (ED). The ED algorithm is based on the method of Lagrangian multipliers using an iterative solution to minimize the cost of delivered power.

$S(i, j) \quad$ transition cost from combination $i$ at hour $k$ to combination $j$ at hour $k+1$. From the source

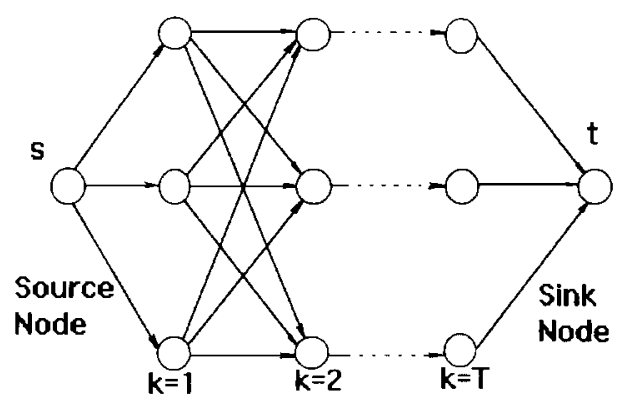

Figure 2. Unit commitment network 
node to the node of the first stage $F(i)=0$, and from nodes of the final stage to the sink node, $S(i, j)=0$.

The arc cost is physically interpreted as the arc length and the unit commitment problem can be stated as a shortest path problem. That is to find the shortest path from source node $s$ to sink node $t$.

\section{IV.2 Solution method}

Now, the unit commitment problem can be solved by a network programming algorithm. The unit commitment network has two important characteristics:

(1) all arc costs are positive, and

(2) only forward arcs are admissible.

So, Dijkstra's method ${ }^{10}$ is an efficient algorithm to solve this problem. The objective of unit commitment becomes:

$$
\begin{aligned}
& \min \sum a(i, j) \\
& a(i, j)=F(i)+S(i, j)
\end{aligned}
$$

subject to constraints (3)-(7).

The Dijkstra's method uses $L(j)$ to store the distance from node $s$ to its associate node $j$ and $P B(j)$ to store the back pointer. Dijkstra's algorithm takes the following steps:

\section{Step 1}

(INITIAL) Set $L(i)=R$ (Some very large number) for all nodes except $s$. Set all back pointers equal to zero. Define the node set $S=\{s\}$. Let $i=s, L(s)=0$.

\section{Step 2}

(FORWARD) For each admissible forward arc originating at node $i$, calculate the length of path to node $j$ through node $i, L(i)+a(i, j)$. If this value is less than $L(j)$, replace $L(j)$ by $L(i)+a(i, j)$. Replace the back pointer for $j$ with $i$.

Step 3

(SELECT) Find $D=\min L(j)$. Let $I E$ be the node for which the minimum is obtained.

\section{Step 4}

(ADD) If $D<R$ include $I E$ in $S$. If $I E=t$ stop with the shortest path from $s$ to $t$. If $I E \neq t$, let $i:=I E$ and go to step 2.

\section{IV.3 Solution procedure}

The procedure used in solving the problem consists of the following steps. It is illustrated by the flow chart in Figure 3.

\section{Step I}

Read in unit data, load demand profiles and spinning reserve requirements.

\section{Step 2}

Generating unit commitment network. To reduce the execution time and the memory required for storage of nodes, unit combination information, a truncated window ${ }^{6}$ and a priority list are used. According to the truncated window, priority list and load demand profiles, a subset of combinations are generated. A combination is represented by a node, and unit commitment network is formed.

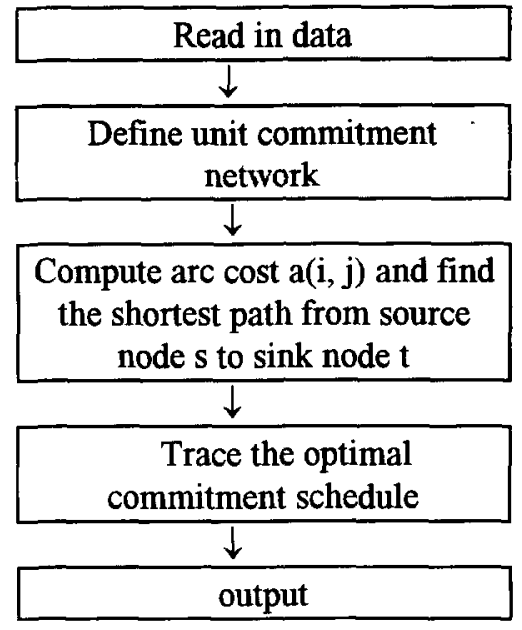

Figure 3. Flow chart of algorithm

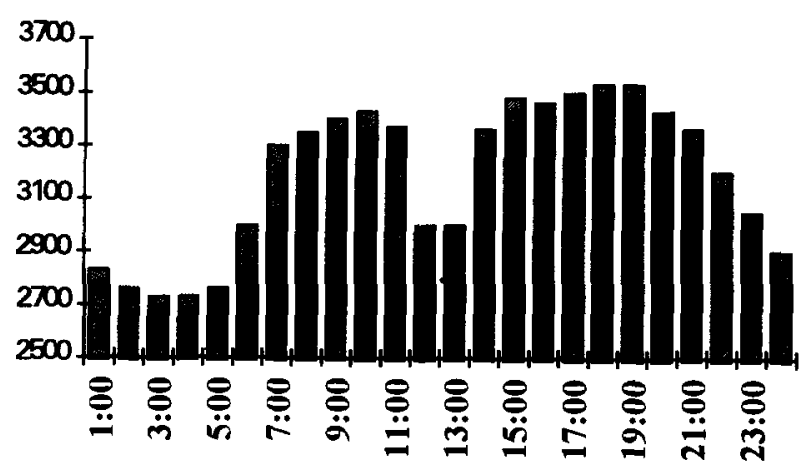

Figure 4. Load profiles

Step 3

Calculating the production cost of these selected combinations. To minimize the overall total cost of unit commitment, the production cost should be minimized. An ED algorithm based on the method of Lagrangian multipliers is used to obtain optimal dispatch.

Step 4

Finding the shortest path from $s$ to $t$. Use Dijkstra's method to find the shortest path. Calculate the arc cost by equation (9). The details of Dijkstra's method have been discussed above.

Step 5

According to the node set $S$, obtain the optimal commitment schedules of all periods.

Step 6

Print out the commitment schedule.

\section{Numerical results}

The procedure outlined above has been programmed on a VAX 11/750 computer, and a realistic Chinese power system is used for an example calculation. This power system has 61 thermal units ranging from $12 \mathrm{MW}$ to $320 \mathrm{MW}$. Unit parameters are given in the Appendix. Forty-three of the 61 units are base load units and are precommitted. The commitment horizons are a 24-hour horizon. The daily system load profiles are shown in Figure 4. In this example, it is assumed that $T_{i}^{\text {on }}$ and $T_{i}^{\text {off }}$ 
Table 1. Results for different search ranges

\begin{tabular}{llll}
\hline Search range & $\begin{array}{l}\text { Operation cost } \\
(1000 \mathrm{~kg} \text { standard coal })\end{array}$ & $\begin{array}{l}\text { Startup cost } \\
(1000 \mathrm{~kg} \text { standard coal })\end{array}$ & $\begin{array}{l}\text { Total system cost } \\
(1000 \mathrm{~kg} \text { standard coal })\end{array}$ \\
\hline 3 & 27342.96 & 89.36 & 27432.32 \\
5 & 27326.13 & 89.86 & 27415.99 \\
7 & 27326.13 & 89.86 & 27415.99 \\
\hline
\end{tabular}

Note: In China, the production cost is defined as $1000 \mathrm{~kg}$ standard coal. When $1000 \mathrm{~kg}$ standard coal burns, it produces $7000 \mathrm{cal}$ of thermal energy.

Table 2. Calculation time and results of different algorithms

\begin{tabular}{lll}
\hline Method & $\begin{array}{l}\text { Computation } \\
\text { time (s) }\end{array}$ & $\begin{array}{l}\text { Total system } \\
\text { cost (1000 kg } \\
\text { standard coal) }\end{array}$ \\
\hline $\begin{array}{l}\text { DP-TC } \\
\text { Network } \\
\text { algorithm }\end{array}$ & 210.42 & 27415.99 \\
\hline
\end{tabular}

are equal to $2 \mathrm{~h}$. The initial conditions of the units are:

$t_{i}^{\text {on }}=48$ hours for units from 1 to 43 ,

$t_{i}^{\text {off }}=-1$ hour for units from 44 to 61 .

For comparison, a procedure based on the DP-TC method $^{5}$ is also programmed on a VAX $11 / 750$ computer. The number of units in the search range for both the proposed method and DP-TC method is 5, the priority lists used by both methods are the same. The results for different search ranges are listed in Table 1 , and the calculation time and results of different algorithms are given in Table 2.

Table 2 illustrates that the proposed algorithm can obtain a solution which is as good as that obtained by the DP-TC method in much less time due to the use of network structures.

\section{Conclusions}

This paper presents a new algorithm for the short-term unit commitment problem. This method formulates the unit commitment problem as a network optimization problem and solves this problem by a network programming technique. The optimization algorithm that exploits network structures can be much faster than general algorithms to produce solutions of the same quality. Moreover, any fuel consumption function and any model of start-up cost can be used in this method.
Results obtained from this study have verified the validity and efficiency of the algorithm and showed the application potential of this method to Chinese power systems. A unit commitment problem with 61 units and 24 periods can be solved in about $45 \mathrm{~s}$.

\section{References}

1 Baldwin, $\mathbf{C} \mathbf{J}$ 'A study of the economic shutdown of generating unit in daily dispatch' AIEE Trans. Power Appar. Syst. Vol PAS-78 (1959) pp 1272-1284

2 Garver, $\mathbf{L} \mathbf{L}$ 'Power generation scheduling by integer programming development of theory' AlEE Trans. Power Appar. Syst. Vol PAS-82 (1963) pp 730-735

3 Muckstadt, J A 'An application of mixed-integer programming duality to scheduling thermal generating system' IEEE Trans. Power Appar. Syst. Vol PAS-87 (1968) pp 1968-1978

4 Lowery, $\mathbf{P}$ G 'Generating unit commitment by dynamic programming' IEEE Trans. Power Appar. Syst. Vol PAS-85 (1966) pp 422-426

5 Pang, $C \mathrm{~K}$ and Chen, $\mathrm{H} \mathrm{C}$ 'Optimal short-term thermal unit commitment' IEEE Trans. Power Appar. Syst. Vol PAS-95 (1976) pp 1336-1346

6 Cohen, A I and Yoshimura, M 'A branch-bound algorithm for unit commitment' IEEE Trans. Power Appar. Syst. Vol PAS-102 No 2 (1982)

7 Van den Bosch, P P J and Honderd, G 'A solution of the unit commitment problem via decomposition and dynamic programming' IEEE Trans. Power Appar. Syst. Vol PAS-104 (1985) pp 1684-1690

8 Ouyang, $\mathbf{Z}$ and Shahidehpour, $\mathbf{S} \mathbf{M}$ 'A multi-stage intelligent system for unit commitment' IEEE Trans. Power Syst. Vol 7 No 2 (1992) pp 639-645

9 Li, S, Shahidehpour, S M and Wang, C'Promoting the application of expert system in short-term unit commitment' IEEE Trans. Power Syst. Vol 8 No 1 (1993) pp 286-292

10 Jensen, $\mathbf{P} \mathbf{A}$ and Wesley Barnes, $\mathbf{J}$ Network flow programming Wiley (1980) 
Appendix

Table A1. The unit data

\begin{tabular}{|c|c|c|c|c|c|c|c|}
\hline & $\begin{array}{l}a \\
(1000 \mathrm{~kg} / \\
\left.(\mathrm{MW})^{2}\right)\end{array}$ & $\begin{array}{l}b \\
(1000 \mathrm{~kg} / \mathrm{MW})\end{array}$ & $\begin{array}{l}c \\
(1000 \mathrm{~kg})\end{array}$ & $\begin{array}{l}\text { Maximum } \\
\text { generation } \\
\text { (MW) }\end{array}$ & $\begin{array}{l}\text { Minimum } \\
\text { generation } \\
\text { (MW) }\end{array}$ & $\begin{array}{l}S_{0} \\
(1000 \mathrm{~kg})\end{array}$ & $\begin{array}{l}\tau \\
(\mathrm{h})\end{array}$ \\
\hline 1 & 4.987 & 0.233 & 0.00063 & 100 & 100 & & \\
\hline 2 & 7.299 & 0.207 & 0.00081 & 70 & 70 & & \\
\hline 3 & 7.299 & 0.207 & 0.00081 & 70 & 70 & & \\
\hline 4 & 2.072 & 0.113 & 0.0191 & 12 & 9 & & \\
\hline 5 & 2.072 & 0.113 & 0.0191 & 12 & 9 & & \\
\hline 6 & 0.772 & 0.445 & 0.027 & 13 & 10 & & \\
\hline 7 & 1.977 & 0.403 & 0.027 & 24 & 18 & & \\
\hline 8 & 5.318 & 0.074 & 0.09 & 25 & 20 & & \\
\hline 9 & 5.318 & 0.074 & 0.09 & 25 & 20 & & \\
\hline 10 & 5.318 & 0.074 & 0.09 & 25 & 20 & & \\
\hline 11 & 5.318 & 0.074 & 0.09 & 25 & 20 & & \\
\hline 12 & 4.399 & -0.312 & 0.0357 & 15 & 12 & & \\
\hline 13 & 5.044 & 0.189 & 0.046 & 25 & 20 & & \\
\hline 14 & 5.044 & 0.189 & 0.046 & 25 & 20 & & \\
\hline 15 & 23.147 & -1.337 & 0.0343 & 30 & 24 & & \\
\hline 16 & 7.624 & 0.088 & 0.0034 & 50 & 40 & & \\
\hline 17 & 1.498 & 0.344 & 0.0004 & 50 & 35 & & \\
\hline 18 & 1.498 & 0.344 & 0.0004 & 50 & 35 & & \\
\hline 19 & 1.498 & 0.344 & 0.0004 & 50 & 35 & & \\
\hline 20 & 2.036 & 0.3132 & 0.00067 & 50 & 35 & & \\
\hline 21 & 2.036 & 0.3132 & 0.00067 & 50 & 35 & & \\
\hline 22 & 2.036 & 0.3132 & 0.00067 & 50 & 35 & & \\
\hline 23 & 2.036 & 0.3132 & 0.00067 & 50 & 35 & & \\
\hline 24 & 2.036 & 0.3132 & 0.00067 & 50 & 35 & & \\
\hline 25 & 2.036 & 0.3132 & 0.00067 & 50 & 35 & & \\
\hline 26 & 2.575 & 0.2414 & 0.0016 & 50 & 35 & & \\
\hline 27 & 5.456 & 0.2118 & 0.0011 & 60 & 50 & & \\
\hline 28 & 5.456 & 0.2118 & 0.0011 & 60 & 50 & & \\
\hline 29 & 2.575 & 0.2414 & 0.0016 & 50 & 35 & & \\
\hline 30 & 2.575 & 0.2414 & 0.0016 & 50 & 35 & & \\
\hline 31 & 4.978 & 0.2423 & 0.00063 & 110 & 70 & & \\
\hline 32 & 4.978 & 0.2423 & 0.00063 & 110 & 70 & & \\
\hline 33 & 4.978 & 0.2423 & 0.00063 & 110 & 70 & & \\
\hline 34 & 4.978 & 0.2423 & 0.00063 & 110 & 70 & & \\
\hline 35 & 4.978 & 0.2423 & 0.00063 & 110 & 70 & & \\
\hline 36 & 4.978 & 0.2423 & 0.00063 & 110 & 70 & & \\
\hline 37 & 4.936 & 0.2368 & 0.00055 & 100 & 70 & & \\
\hline 38 & 7.889 & 0.1945 & 0.00055 & 120 & 88 & & \\
\hline 39 & 2.31 & 0.285 & 0.00012 & 125 & 100 & & \\
\hline 40 & 4.327 & 0.259 & 0.00012 & 200 & 140 & & \\
\hline 41 & 6.3 & 0.265 & 0.00006 & 250 & 200 & & \\
\hline 42 & 6.3 & 0.265 & 0.00006 & 250 & 200 & & \\
\hline 43 & 6.49 & 0.259 & 0.00005 & 320 & 150 & & \\
\hline 44 & 2.575 & 0.241 & 0.0016 & 50 & 35 & 24.62 & 29 \\
\hline 45 & 2.306 & 0.313 & 0.00067 & 50 & 35 & 24.62 & 29 \\
\hline 46 & 7.624 & 0.088 & 0.0034 & 50 & 40 & 26.42 & 29 \\
\hline 47 & 2.776 & 0.209 & 0.0059 & 25 & 20 & 15 & 20 \\
\hline 48 & 4.399 & -0.312 & 0.0357 & 15 & 12 & 11 & 18 \\
\hline 49 & 2.072 & 0.113 & 0.0191 & 12 & 9 & 8 & 17 \\
\hline 50 & 2.072 & 0.113 & 0.0191 & 12 & 9 & 8 & 17 \\
\hline 51 & 2.072 & 0.113 & 0.0191 & 12 & 9 & 8 & 17 \\
\hline 52 & 5.318 & 0.075 & 0.09 & 25 & 20 & 15 & 20 \\
\hline 53 & 5.318 & 0.075 & 0.09 & 25 & 20 & 15 & 20 \\
\hline 54 & 5.318 & 0.075 & 0.09 & 25 & 20 & 15 & 20 \\
\hline 55 & 4.11 & 0.163 & 0.008 & 24 & 20 & 8 & 17 \\
\hline 56 & 1.6 & 0.228 & 0.0141 & 12 & 10 & 8 & 17 \\
\hline 57 & 1.6 & 0.228 & 0.141 & 12 & 10 & 15 & 20 \\
\hline 58 & 7.95 & 0.395 & 0.0044 & 20 & 5 & 0.6 & 0.2 \\
\hline 59 & 7.95 & 0.395 & 0.0044 & 20 & 5 & 0.6 & 0.2 \\
\hline 60 & 7.95 & 0.395 & 0.0044 & 20 & 5 & 0.6 & 0.2 \\
\hline 61 & 7.95 & 0.395 & 0.0044 & 20 & 5 & 0.6 & 0.2 \\
\hline
\end{tabular}

